\title{
Entre la vida cotidiana y las transformaciones del trabajo
}

\author{
Antonio M artín Artiles \\ Fausto M iguélez \\ Universitat Autònoma de Barcelona. D epartament de Sociologia \\ 08193 Bellaterra (Barcelona). Spain
}

Resumen

El artículo presenta la línea de investigación del Grupo de Esatudios sobre T rabajo y Vida cotidiana, en su contenido, proyección política y metodología pluridimensional. Se pre sentan algunos objetivos prioritarios de las investigaciones llevadas a cabo, tanto sus aspectos teóricos y metodológico-técnicos, como sus resultados. (Redacción)

\section{Abstract}

The article shows the research done by the Study Group on Labour and Everiday life, the content, political endeavour and pluridimensional methodology. Somo prior objectives of several pieces of research undertaken, then theoretical amb methodological aspects as well as their results. (Editors)

\section{Introducción}

El tema que presentamos se refiere a la línea de investigación sobre el trabajo que ha sido realizada por parte de un grupo (QUIT) de profesores, ayudantes y becarios del Departamento de Sociología a lo largo de los últimos diez años. En ella han intervenido a veces personas de otros ámbitos de las ciencias sociales.

La investigación del grupo ha tenido al gunas características que quisiéramos resaltar, porque probablemente la han condicionado. La primera tiene que ver con el contenido. Se ha tratado de investigar el trabajo en su proyección «hacia afuera», es decir, hacia lo que hemos llamado la vida cotidiana; el trabajo productivo no se puede entender si no es en relación con el trabajo doméstico y con las actividades e inactividades del no trabajo, lo que nos ha llevado siempre a hablar también de actitudes, valores y representaciones. Se ha querido, por tanto, romper la sectorialización y la hiperespecialización que tradicionalmente ha caracterizado la investigación en ciencias sociales - quizá una de las razones de su esterilidad- y de volver a una cierta globalización de los problemas, lo que, en principio, no debería impedir su concreción. 
La segunda característica tiene que ver con la proyección política de la investigación. La preocupación no ha sido la de encontrar «recetas» para solucionar los problemas prácticos que nuestra sociedad tiene en el trabajo - paro, representatividad, jerarquización, desigualdad- , sino partir de esos problemas prácticos para buscar algún tipo de explicación que permitiera entenderlos mejor en su complejidad para luego poder actuar. Como no somos indiferentes a que se actúa de cualquier manera, hemos buscado determinado tipo de «clientes» y buscado posibles salidas a los problemas, salidas que no eran indiferentes.

En tercer lugar, se ha hecho servir una metodología pluridimensional. $\mathrm{H}$ emos entendido que las metodologías eran caminos y por tanto hemos utilizado todos los que estaban a nuestro al cance. Además, hemos querido experimentar otros, dedicando en bastantes de nuestras investigaciones tiempo, esfuerzo e imaginación a experimentar y aplicar nuevas propuestas metodológicas y nuevas técnicas de búsqueda y tratamiento de la información.

En orden a sistematizar el tratamiento de la actividad investigadora en este campo, quisiéramos diferenciar una doble temática: por un lado, las transformaciones del trabajo y su repercusión sobre las condiciones de vida, por otro, el trabajo entendido como relación social en sí. Esta distinción no necesaria mente se ha traducido en investigaciones diferenciadas, a veces una misma investigación ha abordado ambas problemáticas (además de otras de rango menor); a veces la investigación concreta se ha centrado sobre una de ellas. $C$ on todo, indicaremos en cada una de las partes que se describen a continuación una serie de investigaciones concretas como más decantadas hacia una u otra temática.

\section{Transformaciones del trabajo y condiciones de vida}

\section{a. El objetivo general de esta temática de investigación}

El objetivo ha sido el de entender las transformaciones que están teniendo lugar en el trabajo y, con ello, dotarnos de algunos elementos de políticas reguladoras 0 anticipadoras o correctoras de esas transformaciones. H a sido ésta una temática vigente en la investigación desde que se sospecha que entra en grandes cambios un modelo al tamente consolidado, el fordismo. La reflexión sobre la transformación se centra en el trabajo mismo (Piore, Sabel, 1990), en su condicionamiento de la actividad humana (Baverman, 1975) o en su repercusión sobre la identidad del ser social y, con ello, sobre la estructura social misma (O ffe, 1990), por citar algunos de los clásicos al respecto.

$\mathrm{N}$ uestro propósito ha sido entrar en el meollo de esa transformación, lo que nos podría permitir entenderla como el contexto que condiciona en buena parte - ¿quizá hoy cada vez menos? - la acción de la persona como ser social, es decir, el tipo de interacción que realiza. Por eso las transformaciones del tra bajo que hemos contemplado han tenido una triple dimensión. Por un lado, hemos estudiado los cambios en sí en la actividad humana que llamamos tra - 
bajo productivo: requisitos previos, actuación requerida, relaciones con la tecnología, posibilidades de traducción en unas o en otras condiciones de vida. A continuación hemos tenido en cuenta los contextos en los que se ha dado dicha tranformación: el contexto político (intervención de la sociedad, a través de alguna forma de regulación), el contexto económico o las presiones del mercado (diversas según la amplitud geográfica del mismo), el contexto legal e institucional. En algún grado esos contextos son también los «recursos» de los que pueden echar mano los actores del trabajo, sea los individuales o los colectivos. Por último, había que tener en cuenta las estrategias que pueden ser estrategias de actores sociales colectivos o bien de actores sociales individuales.

En particular hay unos ejes de transformación del trabajo que tienen una cierta relevancia para cualquier observador. $\mathrm{H}$ ay una transformación interna de la actividad en relación con las capacidades y la fuerza de trabajo requerida; aquí el gran cambio tiene que ver con la fuerte expansión de la llamada actividad de los servicios (a sabiendas de que esta misma terminología puede tener cada vez un significado menos preciso). ¿Presupone esta transformación menor al ienación y más autonomía de la persona? ¿Se limita la importancia del tiempo de trabajo y de la actividad llamada trabajo productivo en la vida de las personas?

Pero la transformación más visible es la quiebra de lo que muchos autores Ilaman la «norma social» del empleo. Es decir, hasta hace pocos años en las sociedades Ilamadas desarrolladas se asumía que la gente que así lo deseaba podía contar con un empleo estable, en un ámbito de actividad en el que se profesionalizaba, ocupándole eso una parte substancial de la jornada (trabajo a tiempo completo), la mayor parte de su vida adulta. Resulta que ahora hay personas que queriéndolo no encuentran empleo o cuando lo encuentran es esporádico, de pocas horas, cambiante. Es un fenómeno que afecta directamente a muchas personas e indirectamente - es decir, a través de parientes 0 amigos - a muchas más. Esto puede estar teniendo consecuencias muy importantes sobre la vida cotidiana de la gente y, en térmìnos colectivos, sobre la cultura (valores, ideas, representaciones). Aquí entraría nuestro particular punto de vista.

A lo largo de estos años hemos estado estudiando estas transformaciones en relación con una serie de factores que para nosotros no sólo son los factores explicativos, sino que en sí mismos configuran una estructura de la sociedad bastante distinta de la de hace tres o cuatro décadas. Entre los mismos cabe referirse al factor político - democratización en nuestro país, entrada en la Unión Europea- , remercantilización - después dela «desmercantilización» (Polanyi, 1989) del período anterior- , lo que en analisis político se traduce por neoliberalismo; también a los cambios económicos (sobre todo, las transformaciones de la empresa - descentral ización, multinacionalización- la desectorialización; también los culturales, en particular los cambios espectaculares en el consumo y en el tiempo de no trabajo; también, y sobre todo, las estrategias de los actores (corporativismo de los sindicatos y organizaciones del trabajo, empresarización de las relaciones laborales, individualización del significado 
del trabajo); también las políticas de empleo: hacer frentea la crisis del empleo con la precarización del mismo como objetivo o bien hacerle frente con un «refortalecimiento» de la norma social del empleo.

\section{b. Puntos de vista teóri cos más relevantes}

H emos huido de un corpus teórico general, quizá porque no hemos sido capaces de producirlo y, por tanto, nuestra «huida» ha sido una forma de defensa.

Por el contrario, hemos echado mano de perspectivas teóricas sectoriales, que tenían el objetivo de orientar la investigación y de dotarnos de conceptos, indicadores y clasificaciones adecuadas.

$\mathrm{H}$ ay una de estas perspectivas que ha tenido una importancia superior a las demás: la perspectiva de la vida cotidiana. $\mathrm{H}$ emos partido de concebir $\mathrm{el}$ trabajo humano como un todo (el que se realiza en el mercado, el doméstico), que es la actividad que nos relaciona con la naturaleza y con otros en vistas a producir y transformar lo necesario para vivir y vivir cada vez mejor. (Sobre esto, véase la forma en la que nosotros hemos entendido la teoría de la producción/reproducción en otra de las ponencias pertenecientes a este mismo grupo que se presenta en estas jornadas.) Pero como ese trabajo en su plasmación formal está transido por una doble división, la división social y la división sexual, que lo sitúa aparentemte en «dos campos», con significados sociales distintos, lo que se ha buscado ha sido el puente entre ambos, que para nosotros ha sido la vida cotidiana.

Bajo tal advocación no hemos entendido ni los intersticios de las demás actividades, ni lo rutinario ni lo anodino, ni la privacidad ni lo impenetrable a lo social. Por vida cotidiana hemos entendido, en primer lugar, una cierta situación de tensión de las personas, es decir, la capacidad de los actores socia les de actuar sobre las estructuras que los envuelven al tiempo que están «constreñidos» por ellas (tengo que trabajar al tiempo que intento que ese trabajo me resulte lo más satisfactorio posible). En segundo lugar, hemos entendido por vida cotidiana una cierta capacidad que tienen las personas de estructu rar (es decir, organizar, jerarquizar, dosificar, maximizar, minimizar) las diversas actividades de que se compone la vida todo a lo largo de ella, por más que estamos en un sistema social que quiere apropiársenos esa capacidad estructuradora - 0 donde algunos intentan, y logran, estructurar la vida de otros- , algo de dicha capacidad siempre queda y sobre todo queda el instinto, el deseo, la ilusión de incrementar esa autonomía, y algo siempre se logra, si es menos en una actividad es más en otra, si está minorizado en unos días o meses puede estar maximizado en otros. Tensión y estructuración no han de ser entendidas únicamente en el nivel factual o de las prácticas materiales, sino también en el nivel de las representaciones, simbologías y actitudes siendo este ámbito una fuente de posibles transformaciones sobre la vida de uno mismo o las vidas de los demás. Es decir, nos estamos refiriendo a la coherencia que uno puede dar a la propia vida o a las incoherencias con las que tiene que o quie re vivir. 
Resumiendo (véase QUIT, 1998), uno puede ser hombre o mujer, viejo, maduro 0 joven, de clase trabajadora, media 0 alta, y esto, aunque condiciona no predetermina su vida cotidiana. $\mathrm{H}$ ay un importante margen de posibilidades y, sobre todo, hay la esperanza de incrementarlas. El trabajo (en su globalidad) y su relación con el no trabajo tiene un papel importante en las posibles variaciones a esa supuesta predeterminación.

También hemos asumido la perspectiva de la segmentación del trabajo productivo. $\mathrm{H}$ ay diversos autores que la explican. Aunque fieles a nuestros propósitos, también desde ahí hemos intentado pasar a ver qué significa eso en la otra parte del trabajo (el doméstico) y en el conjunto de la vida de las personas. Es decir, la segmentación ayuda a entender la posición de poder-subordinación también en el ámbito del trabajo reproductivo y del no trabajo (tenerno tener, más importancia-menos importancia, tiempo de no trabajo más autónomo o más heterónomo). Aunque nunca en forma mecánica ni prefija da (véase apartado sobre vida cotidiana).

\section{Aspectos técnico-metodológicos}

$\mathrm{H}$ ay algunos aspectos previos a entrar en el debate de la metodología en cuanto tal, válidos tanto para este ámbito como para otros en los que interviene el trabajo de grupo como tal, pero que es necesario señalar ahora por cuanto influyen fuertemente en la metodología.

El primero es el de la negociación. El grupo mantiene un proceso constante de negociación en su interior, tanto por lo que respecta a la definición de los objetivos a al canzar cuanto en lo tocante a la perspectiva desde la que se abordan (teoría) y a los instrumentos que se utilizan para examinar los «hechos». D icha negociación busca el máximo consenso posible y, por tanto, «gasta» muchísimo tiempo y recursos en cuestiones de procedimiento. 0 bviamente, negociación significa posibilidad de diversos puntos de vista y consenso supone un equilibrio en un momento determinado. $\mathrm{N}$ i una cosa ni la otra suponen que todos los puntos de vista y todos sus promotores tienen la misma fuerza, pero sí que son explicitados y defendidos con el mismo instrumento: la argumentación.

El segundo es la relación con el cliente (sea éste la Administración, otra institución, una organización privada como otros equipos o un sindicato). En este aspecto, el cliente puede hacer su demanda, pero la definición operativa de la misma en términos de intereses de investigación es realizada por el grupo. C on todo, en la mayoría de los casos la investigación ha partido de peticiones del grupo realizadas a instituciones públicas (M inisterio de Educación, Comisión Europea, G eneralitat, etc.), lo que facilita mucho dicha relación con el cliente.

Entrando en los aspectos más directamente técnico-metodológicos, cabría referirse, en primer lugar, a la multidimensionalidad de la metodología utilizada. Esto significa que la multidimensionalidad de los fenómenos estudiados no puede ser aprehendida sino sobre la base de una semejante multiplicidad 
de instrumentos de captación y val oración. Pero también quiere decir que esos diversos instrumentos se tienen que contrastar, y en cierto modo relativizar, unos con otros. Así, el cuestionario tiene que ser contrastado con la entrevista, la entrevista con la observación, las estadísticas con el anál isis del discurso.

H emos dado un papel preminente a la observación estructurada. U na observación que nos permite captar qué es lo que hacen los diversos actores sociales bajo diferentes circunstancias. Esta técnica da una alta posibilidad al actor para actuar sin coacción al tiempo que permite al observador sistematizar dicha experiencia gracias a una cierta estructuración de la observación.

Un segundo aspecto de gran importancia ha sido la entrevista semiguiada y su interpretación. D ebido al carácter globalizador que hemos dado al estudio sobre el trabajo, el tipo de entrevista semiguiada que hemos realizad o ha tenido siempre un marcado carácter biográfico, lo que posibilita tener en cuenta la relación buscada entre trabajo y vida cotidiana. En efecto, con dicho instrumento el trabajo se puede relacionar con la familia, los estudios, las experiencias personal es, las relaciones con otras personas, la concepción del tiempo y del trabajo (productivo y reproductivo), del consumo y del tiempo libre. Al análisis e interpretación de dichas entrevistas, realizadas por el conjunto del equipo y por subequipos de manera coordinada, se ha dedicado mucho tiempo y muchos esfuerzos en el proceso investigador. En realidad ese análisis e interpretación han posibilitado un importante debate en el grupo, tanto sobre los fenómenos que la entrevista recogía cuanto sobre los conceptos y la teoría necesarios para interpretarlos. $\mathrm{H}$ a sido una de las actividades de mayor cohesión del grupo de investigación.

El grupo ha tenido siempre una preocupación muy relevante por experimentar nuevos instrumentos técnico-metodológicos, para captar dimensiones significativas de los fenómenos estudiados, como se decía en la introducción. Por ello ha experimentado el análisis consensual de los textos obtenidos a través de entrevista, ha aplicado el análisis de redes, etc.

\section{d. Algunos resultados}

Estos diez años de investigación han permitido captar que tiene sentido el estudio del trabajo desde una perspectiva más amplia, compleja y pluriforme que llamamosla vida cotidiana. Ello nos ha permitido comprender mejor el papel que el trabajo en toda su globalidad (productivo, doméstico y voluntario) tiene en la vida de las personas. T ambién el tiempo y su distribución han pasado a tener un papel relevante en la explicación de la acción y la interacción huma nas. Esto es válido por lo que se refiere a la materialidad del actuar humano (lo que se hace, la experiencia, la materialidad de las actuaciones), pero también por lo que toca a las actitudes (predisposición a actuar en una determinada dirección) y a las representaciones (ideas y valores que dan sentido a la actua ción humana).

Todo esto nos ha llevado paulatinamente a una nueva fase en la que definimos las relaciones sociales como menos materialistas - aun sin olvidar el 
aspecto clave del materialismo - y como menos determinadas por el trabajo productivo, sino también por otros aspectos claves de la vida que no son el trabajo productivo, aunque se puedan relacionar con él.

La investigación realizada ha permitido profundizar mucho más en las repercusiones sociales y sociológicas de la transformación del trabajo. Significado del empleo precario y del paro, transformaciones del mercado de trabajo (de los intereses y objetivos que lo animan), tanto en la empresa como en el sector 0 en un determinado territorio.

También hemos podido entender mejor y apreciar con mayor precisión las consecuencias efectivas sobre la vida concreta de las personas de la división sexual del trabajo. Al tiempo que se han captado y propuesto al gunas líneas para disminuir las consecuencias negativas de tal división.

H emos podido entrar más a fondo en lo que significa hoy la dualización del trabajo productivo y sus consecuencias posibles sobre la dualidad social. Diversas investigaciones han sido utilizadas en asignaturas como Estructura Social, Sociología del Trabajo, Sociologia de la Empresa.

Un resultado importante tiene que ver con el input que la investigación nos ha permitido introducir en diversas asignaturas de técnicas de investigación social. D iversos doctorados se han basado en investigaciones realizadas. En tal sentido, pensamos que la investigación ha contribuido a la tarea formativa de los estudiantes, tanto en diplomaturas y licenciaturas como en terceros ciclos.

Por último, habría que señalar que muchas de las investigaciones han sido publicadas. D e lo que se podría esperar que alguna de ellas, en algún aspecto, haya servido a al guien para comprender mejor ciertos fenómenos o poder intervenir más en otros.

\section{Política de recursos humanos}

\section{a. El objetivo general de esta temática de investigación}

Esta segunda línea de investigación, desarrollada entre 1992 y 1998, surge en el contexto de la crisis del modelo de regulación laboral keynesiano-fordista. D esde los años ochenta la crisis de este modelo ha venido propiciando la introducción de nuevas formas de regulación laboral descentralizada, microcorporativista y empresarializada, que paralelamente también han puesto en crisis al modelo tradicional de sindicalismo. En contrapunto, una de las formas de expresión de estas nuevas tendencias emergentes son las Ilamadas políticas de «recursos humanos» (RRH H , en adelante), inspirada teóricamente por una especie de funcionalismo tosco, junto con otros ingredientes procedentes de las teorías motivacionales (desde el ámbito de la psicosociología).

D e ahí nuestro interés académico por conocer el discurso y las prácticas de las «políticas de recursos humanos». Así, en términos concretos, esta línea de investigación se inició fundamentalmente a partir del estudio sobre el impacto que ha tenido la preparación de los Juegos 0 límpicos de Barcelona en el ámbito 
laboral, tecnológico y organizativo de las empresas de área metropolitana de Barcelona; este estudio fue encargado por el $\mathrm{COOB}^{1}$. Posteriormente, esta línea de investigación se continuó desarrollando en base a tres estudios más: dos estudios comparados en el ámbito europeo. El primero, en colaboración con el IRES de L ombardía, sobre las políticas de recursos humanos en cuatro regiones motoras de Europa ${ }^{2}$, el segundo estudio, relativo a las políticas de participación directa e implicación en el trabajo en el ámbito europeo, encargado por la European Fondation for W orking C onditions ${ }^{3}$. Final mente, esta línea concluye con un tercer estudio, financiado por la D G ICYT ${ }^{4}$, sobre la formación en el trabajo.

\section{a. 0 bjetivos específicos}

Los objetivos de esta línea de investigación han consistido en analizar las políticas de recursos humanos en las empresas. En este sentido, definimos como política de recursos humanos al discurso empresarial que sostiene la identidad común entre capital y trabajo, que sustenta la idea de la existencia de valores compartidos en torno a una comunidad de intereses. M ientras que por prácticas de recursos humanos entendemos el conjunto de medios a través de los cua les las empresas recitan, motivan, promocionan, recualifican y controlan a sus empleados. En las distintas investigaciones hemos desagregado este concepto en las siguientes dimensiones:

- discurso integrador;

- estilo y formas de comunicación;

- reclutamiento y selección de la fuerza de trabajo;

- formas de gestión del empleo;

- participación e implicación en el trabajo;

1. El resultado final de esta investigación está publicado en la obra colectiva del G RUP D 'Estudis So ciolò gics SO bre LA VIDA Q uotidiana I el Treball (1997). Economía Trabajo y Empresa. M adrid: CES, p. 314.

2. Este estudio, basado en el método de casos, se llevó a cabo en Lombardía, BadenW ürttemberg, RhôneAlps y Catalunya. Está publicado por REGINI, M arino (a cura, 1996). La formazione delle risorse umane. Bolonia: II M ulino. Vease también REGINI; BAH N M ÜLLER (1997). Bet Practice oder funktionale Aquivalenz? M unchen und M ering: Rainer H ampp Verlag.

3. Esta investigación ha sido fundamentalmente a partir de la bibliografía especializada, a fin de formular un «estado de la cuestión». Está publicada en Frö LICH; PELKRUH (1996). Direct participation and Organisational Change. Fashionable but M isunderstood? D ublín: European Fondation.

También otra parte dela citada investigación sellevó a cabo mediante entrevistas a los agentes sociales; veáse, en este sentido, Reg ALIA, I da (1995). H umanize W ork and Increase Pro fitability? D ublín: European Fondation.

4. Algunas partes de este estudio han sido publicadas, véase Lope; M artín Artiles (1995). «Las relaciones entre formación y empleo». M adrid: Revista Economía y Sociología del Trabajo, n²7/28, así como, en próxima aparición, M artín Artiles, A; Lope, A. (1999). «iSirve la formación para tener empleo»?. Barcelona, RevistaPapers, $n^{\circ} 58$. 
- motivación, incentivos y promoción;

- recualificación, formación y promoción.

\section{b. Aspectos teóricos e hipótesis}

Las políticas de RRH H han tenido un interés creciente en la literatura especializada y en las prácticas empresariales de los años noventa. Por un lado, en términos general es, la extensión de las políticas de RRH H está asociada a la hegemonía del discurso neoliberal, al predominio de la ideología asociada a la «disciplina de mercado» y al declive sindical resgistrado desde la década de los ochenta. En este contexto ideológico se han ido recuperando las ideas de la vieja perspectiva funcionalista que se encuentra en la escuela empresarial «unitarista» ${ }^{5}$. É sta considera que capital y trabajo tienen la misma comunidad de intereses y, por tanto, la esencia de las relaciones laborales es la cooperación y el consenso; mientras quee conflicto es un elemento exógeno a la organización.

Por otro lado, también la extensión de las políticas de RRH H constituye parte de las respuestas empresariales a la agudización de la competencia en los mercados de productos. Esto hallevado a las empresas a buscar elementos diferenciadores y competitivos a través de la calidad y la diversificación de los productos; adoptar nuevas tecnologías y nuevas formas de organización del trabajo. D e ahí la búsqueda de la cooperación, implicación y participación en el trabajo para optimizar el rendimiento de los nuevos equipos técnicos y los nue vos métodos.

Por consiguiente, la búsqueda de la cooperación entre capital y trabajo, subyacenteen el discurso de las políticas de RRH H , ha contribuido a configurar un discurso «microcorporativo», de «espíritu de empresa», basado en la idea de comunidad de intereses, en el fomento de actitudes proorganizativas como método de cohesión interna y el desarrollo de una serie de valores éticos y morales integradores y compartidos, lo que tiene a la postre como finalidad la construcción de una identidad colectiva. Es decir, setrata de un discurso integrador que esconde el conflicto de intereses entre capital y trabajo, al tiempo que relega a un segundo plano a las formas de representación colectiva y sindical.

En definitiva, este enfoque «unitarista», en cierto modo subyacente en la política de recursos humanos, trata de enfatizar los elementos de integración,

5. D e hecho, la revitalización de esta escuela empresarial «unitarista» y del viejo paradigma funcionalista se debe leer en claves ideológicas, propagandísticas y de discurso dominante asociado a la hegemonía neoliberal. 0 como dice Watson (1995), en el fondo no es hoy propiamente un enfoque académico. De hecho, desde el ámbito académico la síntesis neofuncionalista (vease D ahrendorf (1990) y Rex (1985) sobre el conflicto y la cooperación superan con mucho aquél tosco funcionalismo. También en esta línea relativa al carácter dual y contradictorio de las relaciones laborales, como relaciones de conflicto y pacto, han contribuido otros autores como H yman (1981) y Poole (1991). 
cooperación, participación, implicación y motivación para obtener prestaciones de calidad y flexibilidad de la fuerza de trabajo, así como a los requerimientos de la innovación tecnológica y a las nuevas formas de organización del trabajo. En otras palabras, la organización del trabajo postaylorista estaría dejando de considerar la fuerza de trabajo como un mero coste para ser contemplada como un recurso a desarrollar. Pero también, al mismo tiempo encierra un fuerte discurso antisindical, lo que se expresa mediante prácticas tendentes a una cierta individualización de las relaciones laborales para quebrar la capa cidad de acción colectiva del sindicalismo.

Este discurso integrador, que pretende desarrollar el potencial de los recursos humanos, no parece - en términos de prácticas- extensible a toda la gestión de la fuerza de trabajo.

Por el contrario, nuestra hipótesis consiste en apuntar que las prácticas de gestión empresarial siguen criterios selectivosy disgregadores. La gestión empresarial de los RRH H tienden a distinguir entre una gestión integradora y proorganizativa, ubicada en lo que podemos llamar «núcleo organizativo», compuesto por empleados, oficiales y cuadros técnicos cualificados, que tienen un tratamiento salarial y profesional distinto. Q uienes componen este «núcleo» tienen acceso a la formación, recualificación, promoción interna y acceso a los incentivos materiales y simbólicos, incluso tienden a tener relaciones laborales individualizadas.

$Y$, por otro lado, en la «periferia», constituida por la fuerza de trabajo con bajas cualificaciones, los criterios de gestión de la fuerza de trabajo se plantean bajo la «ógica de la disciplina de mercado», no en términos de integración, sino de flexibilidad, rotación y externalización. De ahí que no accedan a los circuitos de formación y reciclaje, ni al circuito de recocimientos simbólicos. No obstante, la mayor o menor presencia de los sindicatos condiciona o limita estas políticas de gestión de los recursos humanos. En este sentido, también sostenemos la hipótesis de que el sindicalismo - o la misma agrupación colectiva que pueden desarrollar los «grupos de referencia»- sigue siendo funcional al sistema organizativo de empresa, al menos para ciertos segmentos de empleo cuyo tratamiento y gestión de las condiciones de trabajo continúan siendo homogéneos.

\section{c. Aspectos metodológi cos y técni cos}

Estas investigaciones se han Ilevado a cabo a partir de una aproximación cuantitativa y otra cualitativa al objeto de estudio. En primer lugar, la aproximación cuantitativa tenía como finalidad mesurar el grado de extensión de las políticas de recursos humanos, así como el grado de modernización tecnológica y organizativa de las empresas de la R egión M etropolitana de Barcelona6. Asi-

6. En este sentido, se realizó una encuesta a una muestra de 649 empresas de más de 10 trabajadores. 
mismo, la muestra fue estratificada en base a dos criterios: uno, a partir del tamaño de la empresa según su número de trabajadores, y dos, en función de la distribución de los establecimientos en el territorio. La encuesta, realizada para el estudio del COOB, además de una información general y panorámica, nos proporcionó una importante base de datos para posteriores y sucesivas investigaciones.

En segundo lugar, la aproximación cualitativa, diseñada para el mismo estudio, se ha basado en el estudio de casos de empresas, con la finalidad de conocer en profundidad las estrategias empresariales, conocer los significados y los aspectos dinámicos de las políticas de recursos humanos. L os casos estudiados fueron una selección de empresas industrial es y de servicios extraídas a partir de la muestra ${ }^{7}$. En los estudios de casos se han utilizado las técnicas de las entrevistas y los grupos de discusión. Las entrevistas también se han llevado a cabo de manera estratificada, entrevistando tanto a directivos, cuadros medios y trabajadores o empleados directos de producción. I gualmente, se ha entrevistado a los representantes de los trabajadores.

Las otras investigaciones se han basado en el método cualitativo: sellevaron a cabo a partir del método de casos, pero siempre teniendo en cuenta su selección a partir de los datos de la encuesta del CO O B anteriormente citada. Por tanto, se disponía ya de una información cuantitativa de cada uno de los casos a estudiar. En el caso del estudio de los cuatro motores se estudiaron nueve casos; tres empresas del sector de máquinas-herramientas, tres del sector textil y otras tres del sector financiero, de acuerdo con las hipótesis relativas a diferencias en las formas de gestión laboral a tenor del sector de actividad empre sarial. Y, por último, la investigación sobre la formación y el empleo también se fundamentó en el estudio de casos de dos empresas, un gran hipermercado y una empresa textil, ambas significativas en el ámbito del Vallès 0 ccidental. En estos dos casos, además, se realizó una encuesta a la plantilla de cada una de las empresas. En estos casos, las hipótesis a investigar estaban relacionadas con las cualificaciones actitudinales y comportamentales.

Cabe mencionar que, además de la aproximación cuantitativa y cualitativa, en las investigaciones también se han llevado a cabo estudios contextuales sobre el marco económico y social; lo que ha sido clave para poder interpretar las tendencias demográficas, políticas, sindicales e institucionales, y conocer el sistema educativo y productivo del entorno.

\section{d. Resultadosy condusiones}

Aquí no vamos a dar cuenta exhaustiva de las conclusiones de cada una de las investigaciones por separado; sino de las reflexiones final es acumuladas que se desprenden de esta línea de investigación. Atendiendo a las dimensiones ya citadas del concepto recursos humanos, serían las siguientes:

7. Se seleccionaron 20 empresas, estratificadas en dos grupos, entre 100 y 500 y otro grupo de más de 500 trabajadores. 


\section{Factores contingentes}

En primer lugar, cabe señalar los factores contingentes que impulsan o bien dificultan las prácticas de recursos humanos. En este sentido, la renovación en las políticas de gestión de los RRH H se deriva de factores de modernización liga dos con las exigencias de competitividad, los cambios en el mercado, las variaciones en la composición dela demanda y las estrategias de producción flexible de las empresas. Pero también inciden otros factores, tal es como la conflictividad laboral y la presión sindical o, incluso, el cambio en la dirección del departamento de relaciones laborales en favor de un nuevo equipo con criterios modernizantes.

\section{Discurso}

I deología de la integración versus prácticas sel ectivas. En segundo lugar, el discurso de la integración de los trabajadores en el sistema organizativo, basado en la idea de comunidad de intereses, es meramente un enunciado ideológico y formal, muy alejado de las prácticas reales. Las prácticas empresariales siguen, por el contrario, criterios selectivos de inclusión y exclusión.

\section{O rganigrama jerárquico y forma de comunicación}

El organigrama jerárquico se viene transformando: se reducen los escalones de mando a tres o cuatro niveles, al tiempo que se ensancha la base del organigrama. Esta reducción afecta en particular a los mandos intermedios. Las razones de la reducción de los escal ones jerárquicos estriban en la tecnología de la información, que permite recoger información (on-line) sobre el mismo proceso de producción, sin necesidad de filtros intermedios e incluso tomar decisiones rápidas. Por ello, también la comunicación se ha venido convirtiendo en los últimos años en un tema estrella en las políticas de gestión y de formación de los RRH H. Los conocimientos de comunicación y de dinamica de grupos son básicos para el ejercicio del liderazgo en directivos, mandos intermedios y coordinadores de grupos o equipos; los métodos de trabajo y las técnicas de resolución de problemas son requisitos instrumentales para los grupos y equipos de trabajo, tanto de directivos como de trabajadores o empleados (Grup d'Estudis QUIT, 1999).

\section{Reclutamiento, selección y formación}

Los criterios de reclutamiento, selección de personal y exigencias formativas dependen del nivel del puesto de trabajo a ocupar. En este sentido, algunos estudios (Lope, 1994; M artín Artiles, Rebollo, 1997) ponen de relieve que las empresas tienden a contratar cualificaciones de forma polarizada. En otras palabras, las exigencias de cualificaciones real es útiles son tanto cual ificadas como descualificadas; lo que al fin y a la postre está asociado a la división y organización del trabajo dominante.

5. Formación y desarrollo de las cualificaciones en las empresas

La formación es hoy otro aspecto clave en las políticas de personal, tanto desde 
el punto de vista de profesionalización de los trabajadores como para ofrecerles cauces de integración. Pero también la formación parece ser consecuencia de exigencia de los actores social es cuando se encuentran en situaciones de reestructuración tecnológico-organizativa y se aportan experiencias e ilustran con estudios de casos más próximos a la realidad de nuestro entorno, lo que contribuye a la comprensión de la temática para los estudiantes, y para el profesor representa explicar de forma concreta, mediante el método de casos, cuestiones conceptual es y temas abstractos. Asimismo, dicha línea de investigación nos ha posibilitado confrontar bibliografía, dialogar con teorías y estudios procedentes de otras latitudes, que no siempre son generalizables ni transferibles a otros contextos sociales. Por tanto, el vínculo de la investigación con la docencia es en términos de matizaciones y críticas a ciertas teorías o bien de comparación con experiencias de otros países de nuestro entorno.

\section{Bibliografía citada vinculada con el Grup d'Estudis QUIT}

ALÒS; M IGUÉLEZ; RECIO. Transformaciones laborales en la industria dela construcción en Catalunya. Barcelona: CERES-CON C.

FröliCH; DieTER; PEKRUHL; U LRICH (1996). Direct participation and Organisational

Change. Fashi onable but M isundestood? D ublín: European Foundation for the Improvement of Living and Working Conditions, $228 \mathrm{p}$.

GEARY, J.; SISSON, K. (1994). Conceptualising D irect Participation in O rganisational

Change. The EPOC Project. Luxemburgo: $O$ ffice for $O$ fficial Publication of the European Community.

Lo PE, Andreu (1994). Innovació tecnol ògica. Barcelona: Fundació Jaume Bofill, 293 p. Lo Pe, A.; M artín ARTILES, A. (1993). «Cambio técnico y recualificación. Formación y adquisición de las cualificaciones en la empresa. Un estudio de casos. M adrid: Revista Sociología del Trabajo, n 19, p. 69-98.

- (1995). «Las relaciones entre formación y empleo». M adrid: Revista Economía y Sociología del Trabajo, $n^{\circ}$ 27-28, p. 254-266.

Lo Pe, A.; M artín ARtiles, A.; Varella, R. (1994). Literature Study: direct participa tion in organizational change. The case of Spain. Barcelona: G rup d Estudis QUIT. D epartament de Sociologia. UAB.

M artín Artiles, A. (1995). Flexibilidad y relacioneslaborales. M adrid: Consejo Económico y Social, $305 \mathrm{p}$.

M artín ARTiles, A.; G arcía, M . (1992). Los procesos de adquisición de las cualifica ciones en las empresas españolas. Barcelona: ICE-CED EFO P.

M artín ARTILES, A.; LOPE, A. (1994). «D inámica de las cualificaciones y políticas de recursos humanos». M adrid: Revista E conomía y Sociología del Trabajo, n²1/22, p. 115-128.

- (1999). «¿Sirvela formación para tener empleo?» Barcelona: Revista Papers, n 58. M artín Artiles, A.; M IGUÉlez, F.; Lo PE, A. (1998). «H uman resource policies, training and qualifications». Bruselas: Transfer Review, $n^{\circ} 2$. Volumen 4.

M artín ARtiles, A.; M IgUÉlez, F.; PASto R, I. (1996). «C atalogne: le risorse umane en un mercato de lavoro dualistico» [En REGINI, M ARIN 0: La formazione delle risorse umane. Bolonia: II M ulino, 297 p. 
M artín Artiles, A.; Rebollo, O . (1997). Accés dels joves a l'ocupació. Barcelona: Grup Q uit-D epartament de Sociologia de la UAB. Working Paper, $96 \mathrm{p}$.

M IGUÉLEZ, F. y otros (1991). La Encuesta de Relaciones Laborales en Catalunya. Barcelona: CERES-CONC.

- (1996). D esigualtat i canvi. Barcelona: Edicions Proa.

Prieto, CaRlos (1989). «Políticas de mano de obra en las empresas españolas.» M adrid: Revista Sociología del Trabajo, $n^{\circ}$ 6, p. 33-50.

- (1991). «Las prácticas empresariales de gestión de fuerza de trabajo», p.185-211. En M IgUÉleZ; PrIeto: Las relaciones laborales en España. M adrid: Siglo XXI.

QUIT GRUP D'ESTUdIS SOCIOLÒ GICS SOBRE LA VIDA QUOTIDIANA Y EL TREBALL (1997). E conomía, trabajo y empresa. M adrid: Consejo Económico y Social.

- (1998). Treball i vida quotidiana. Barcelona: Revista Papers, $n^{\circ} 55$.

- (1999). ¿M ás formación, más empleo? M adrid: Consejo Económico y Social (en imprenta).

Rebollo, O .; M artín Artiles, A.; M iguélez, F. (1993). El sindicalismo a través de sus protagonistas. Barcelona: CERES-CONC.

\section{O tra bibliografía citada}

Braverm an, H . (1975). Trabajo y capital monopolista. M éxico: Ediciones Era.

Kern, H .; SChum AnN, M . (1988). El fin de la división del trabajo. M adrid: M inisterio de Trabajo, $395 \mathrm{p}$.

M ÉH AUT, P.; D ELCOURT, J. (1993). Le role del'entreprise dans la production des quali fications: effects formateurs del'organization du travail. Berlín: CEDEFOP.

O fFE, C. (1990). La sociedad del trabajo. M adrid: Alianza.

PIORE, M .; SABEL, CH . (1990). La segunda ruptura industrial. M adrid: Alianza.

PO LANY, K. (1989). La gran transformación. M adrid: La Piqueta.

STAN KIEWICZ, F. (comp. 1993). Las estrategias de las empresasfrente a los recursoshuma nos. Buenos Aires: Humánitas. 